Table 2. Partridge and possible predator relationships in three areas that were closely examined during the season.

\begin{tabular}{|c|c|c|c|c|}
\hline & & \begin{tabular}{|l|} 
Gray \\
Partridge \\
\end{tabular} & $\begin{array}{c}\text { Snowy } \\
\text { Owls }\end{array}$ & Others \\
\hline Vanscoy-Delisle-Asquith & Jan. 1 & 105 & 1 & 1 Golden Eagle \\
\hline \multirow[t]{4}{*}{20 miles } & " 19 & 47 & 1.2 & 2 eagles \\
\hline & Fèb. 2 & 149 & 1 & 1 eagle, 1 fox \\
\hline & " 15 & 162 & 2 & 1 Goshawk \\
\hline & Mar. 1 & 60 & 1 & - \\
\hline \multirow{4}{*}{$\begin{array}{l}\text { Allan-Elstow-Clavet: } \\
18 \text { miles }\end{array}$} & Dec. 28 & 8 & 1 & - \\
\hline & Jan. 26 & 0 & 0 & 一 \\
\hline & Feb. 23 & 6 & 0 & 一 \\
\hline & Mar. 30 & 0 & 17 & 一 \\
\hline \multirow{4}{*}{$\begin{array}{l}\text { South of Clavet: } \\
7 \text { miles }\end{array}$} & Jan. 26 & 48 & 1 & - \\
\hline & Feb. 16 & 24 & 2 & 1 fox \\
\hline & Feb. 29 & 33 & 6 & 1 eagle \\
\hline & Mar. 30 & 2 & 0 & 1 Golden Eogle \\
\hline
\end{tabular}

index of abundance from year to year. The requirements might be: (1) A route of at least 40 miles (based on miles-per-owl above) along a road likely to be kept open during the winter. A route that starts and ends near home is ideal. (2) Coverage every two to four weeks from November through April. Routes should be covered during the same part of the day each time and, if possible, under clear skies. (3) Observations made in a consistent manner, e.g., a $360^{\circ}$ scanning of the landscape with binoculars at two-mile intervals and naked-eye observations in between while driving 20 30 m.p.h. The same, preferably two, observers should conduct the survey each time. Record should also be kept of snow conditions.

Such an undertaking might also yield information on the status of upland game birds and other species. As indicated above, it can give a birdwatcher a new perspective of what is usually considered a slack season for birds.

\title{
Twenty-third Annual Saskatchewan Christmas Bird Count, 1964
}

\author{
compiled by Mary Houston, Saskatoon
}

Unfavorable weather during the entire Christmas season seems to have discouraged bird counters this year. Reports were received from only 25 Saskatchewan points (two of these count areas reporting from Kelvington) and only 48 species of birds were seen on count day with an additional seven species during the count period. A Trumpeter Swan at Regina, the only species not seen previously on a Saskatchewan Christmas bird count, brings the 23-year total to 111 species.

Black-billed Magpies, seen in all 25 localities and House Sparrows reported from 23, were the commonest birds, with Black-capped Chickadees running a close third in 19 of the 25 possible localities. Sharp-tailed Grouse were reported in 17 and Gray Partridge in 16 of the counts. Of particular interest was the Horned Lark, seen in 13 localities, many of them further north than is to be expected at this time, especially in a year with below average temperatures and above average snowfall.

Regina led the field with 37 species, including quite a variety of waterfowl. Saskatoon's claim to fame was perhaps endurance-the temperature being $-35^{\circ}$ at 9 a.m. and still $-34^{\circ}$ at noon.

One count each was received from Alberta and the Northwest Territories. 
Details of the Manitoba counts are now being submitted to the Newsletter of the Manitoba Natural History Society.

BANGOR, Sask. Dec. 26; 25 miles by car and around the farm yard. 11 species, 354 individuals. Ruffed Grouse, 1; Sharp-tailed Grouse, 3; Great Horned Owl, 1; Hairy Woodpecker, 2; Downy Woodpecker 1; Black-billed Magpie, 4; Black-capped Chickadee, 4; House Sparrow, 75; Pine Grosbeak, 8; Common Redpoll, 50; Snow Bunting, 200.-Mrs. A. Thompson.

BATTLEFORD, Sask. Dec. 30; 28 miles by car and three on foot in $4 \frac{1}{2}$ hours; temp. $-24^{\circ}$; wind NW at 3 m.p.h.; 22 inches of snow. 12 species, 323 individuals. Ruffed Grouse 2; Sharp-tailed Grouse, 17; Rock Dove, 11; Hairy Woodpecker, 2; Horned Lark, 73; Blue Jay, 1; Black-billed Magpie, 22; Black-capped Chickadee, 3; Bohemian Waxwing, 31; Starling, 1; House Sparrow, 17; Snow Bunting, 143.-Spencer G. Sealy.

BLADWORTH, Sask. Dec. 24; 15 miles by car and around yard; temp. $-30^{\circ}$ to $-15^{\circ}$; calm; 1 foot of loose snow everywhere. 8 species, 390 individuals. Goshawk, 1; Gray Partridge, 22; Horned Lark, 100; Black-billed Magpie, 10; Black-capped Chickadee, 1; House Sparrow, 30; Pine Grosbeak, 1; Snow Bunting, 225. (Add: Sharp-tailed Grouse, 19, Dec. 25 and 3, Dec. 25, and 4, Jan. 2; Rock Dove, 22, Dec. 25; Hairy Woodpecker, 1, Dec. 31; Starling, 2, Jan. 1.)-P. Lawrence Beckie.

DILKE, Sask. Jan. 1, 1965; 5 hours; 36 miles by car, 2 miles on foot and around the farmyard; temp. $-10^{\circ}$ to $6^{\circ}$; wind SW at 5 m.p.h.; 12 inches of snow, drifted. 13 species, 1884 individuals. Goshawk, 1; Golden Eagle, 1; Sharp-tailed Grouse, 12; Gray Partridge, 68; Rock Dove, 16; Snowy Owl, 1; Downy Woodpecker, 1; Horned Lark, 45; Black-billed Magpie, 16; Black-capped Chickadee, 1; Starling, 2; House Sparrow, 220; Snow Bunting, 1500. (Add: Great Horned Owl, '1, Jan. 2.) Boswall Belcher (compiler), Margaret Belcher, Mr. and Mrs. S. R. Belcher, Mrs. J. Mortin.

ELLISBORO, Sask. Dec. 24; 12 miles by car; temp. $-15^{\circ}$; wind light. 5 species, 352 individuals. Sharp-tailed
Grouse, 40; Rock Dove, 8; Black-billed Magpie, 4; House Sparrow, 100; Snow Bunting, 200.-Mrs. P. Churchman.

ERINFERRY, Sask. Dec. 24; at yard feeding station; temp. $-20^{\circ}$; calm. 7 species, 68 individuals. Downy Woodpecker, 1; Gray Jay, 4; Blue Jay, 8; Black-billed Magpie, 1; Black-capped Chickadee, 4; Golden-crowned Kinglet, 30; Snow Bunting, 20.-Mrs. E. A. Dodd.

ETHELTON, Sask. Dec. 29; about yard. 8 species, 324 individuals. Hairy Woodpecker, 2; Downy Woodpecker, 1; Black-billed Magpie, 4; Black-capper Chickadee, 4; House Sparrow, 24; Pine Grosbeak, 12; Common Redpoll, 2; Snow Bunting, 275. (Add: Great Horned Owl, 1, Dec. 23; Raven, 2, Dec. 28.)-Genevieve Belliveau.

FORT QU'APPELLE, Sask. Dec. 26; temp. about $-20^{\circ}$ at noon; about 15 in. snow; 7 species, 114 individuals. Sharp-tailed Grouse, 23; Downy Woodpecker, 1; Black-billed Magpie, 21; Black-capped Chickadee, 4; Bohemian Waxwing, 3; House Sparrow, 46; Snow Bunting, 16. (Add: Ruffed Grouse, 2, Jan. 1; Gray Partridge, 7, Dec. 23, 25; Rock Dove, 7, Dec. 30; Blue Jay, 2, Dec. 28 and 1 Jan. 1, 3; Pine Grosbeak, 7 reports, max. flock of 20; Whitethroated Sparrow, 1, at Callin's feeding station daily from Nov. 16 to Dec. 24, then disappeared.)-Dr. and Mrs. G. D. Barnett, Alden Barnett, E. Manley Callin (compiler), Richard Carter, Mr. and Mrs. Errol Cochrane, Mr. and Mrs. Wm. Gray, Ian Macpherson, Richard Nevard, Horace Reed, S. P. Regan.

GRENFELL, Sask. Dec. 25; 61/2 miles by car and around yard all day; temp. $-10^{\circ}$; wind west at 4 m.p.h.; 10 inches of snow. 5 species, 355 individuals. Great Horned Owl, 1; Horned Lark, 10; Black-billed Magpie, 4; House Sparrow, 250; Snow Bunting, 90. (Add: Sharp-tailed Grouse, 6, Dec. 20; Gray Partridge, 4, Dec. 20; Rock Dove, 5, Dec. 20; Black-capped Chickadee, 1, Dec. 29; Bohemian Waxwing, 17, Dec. 22; Common Redpoll 10, Jan. 1.)-Mr. and Mrs. John Hubbard.

HAWARDEN, Sask. Dec. 24; 20 miles by car and on foot around yard; $21 / 2$ hours; temp. $-25^{\circ}$; cloudy; 1 foot of snow. 5 species, 243 individuals. Gray Partridge, 15; Rock Dove, 10; 
Horned Lark, 13; Black-billed Magpie, 5; House Sparrow, 200. (Add: Sharptailed Grouse, 1, Dec. 21; Snowy Owl, 1, Dec. 20 and 30; Snow Bunting, 1, Dec. 28 and 4, Jan. 1.)-Harold Kvinge.

KELVINGTON, Sask. Dec. 29; around yard throughout the day; 10 inches of snow, but summerfallow bare. 6 species, 92 individuals. Hairy Woodpecker, 2; Downy Woodpecker, 1; Black-billed Magpie, 1; Black-capped Chickadee, 3; Bohemian Waxwing, 55; House Sparrow, 30. (Add: Sharptailed Grouse, 11, Jan. 2; Great Horned Owl, 1, Dec. 23; Raven, 1, Jan. 3; Evening Grosbeak, 5, Dec. 28; Pine Grosbeak, 2, Dec. 28; Snow Bunting, 60, Dec. 30.)-Brian Irving.

KELVINGTON, Sask. Jan. 2, 1965; about the town of Kelvington; one-half hour; temp. $15^{\circ}$ to $20^{\circ}$; variable cloudiness; light wind. 7 species, 51 individuals. Hairy Woodpecker, 1; Down Woodpecker, 1; Blue Jay, 1; Black-billed Magpie, 2; Black-capped Chickadee, 6; House Sparrow, 26; Evening Grosbeak, 14. (Add: Gray Partridge, 6, Dec. 22; Raven, 5, Jan. 3; Pine Grosbeak, 18, Jan. 3.)-Steve Waycheshen.

KINDERSLEY, Sask. Jan. 1, 1965; 8 miles by car and 2 miles on foot; $11 / 2$ hours; temp. $5^{\circ}$; clear; wind, 10 m.p.h. 20 inches of snow. 4 species, 246 individuals. Gray Partridge, 41; Great Horned Owl, 2; Horned Lark, 80; House Sparrow, 123. (Add: Bohemian Waxwing, 5, Dec. 26; Starling, 15, Dec. 29; Snow Bunting, 100, Dec. 28.)- Glen A. Fox (compiler), Kim Lamont, R. A. Lamont.

LUCKY LAKE, Sask. Dec. 29; 95 miles by car; cold, overcast and some snowfall; 20 inches of snow, badly drifted. 6 species, 232 individuals. Gray Partridge, 15; Snowy Owl, 1; Horned Lark, 12; Black-billed Magpie, 2; House Sparrow, 20; Snow Bunting, 182. (Add: Sharp-tailed Grouse, 2, Jan. 2; Rock Dove, 18, Dec. 30; Common Redpoll, 15, on Dec. 25, and 20 on Dec. 26.)-Mrs. E. Boon.

McLEAN, .Sask. Dec. 29; 10 miles by car and around yard; 7 species, 38 individuals. Ruffed Grouse, 2; Gray Partridge, 10; Hairy Woodpecker, 2; Downy Woodpecker, 2; Horned Lark, 8; Black-billed Magpie, 2; Black-cap- per Chickadee, 12. (Add: Bohemian Waxwing, 6, Dec. 22; Pine Grosbeak, 4, Dec. 22.)-Mrs. Harold Bray, Mrs. Hilda Newton.

MOOSE JAW, Sask. Dec. 26; 181/2 party hours and 20 miles on foot and $11 / 2$ party hours and 37 miles by car; temp. $-20^{\circ}$ to $-14^{\circ}$; wind $\mathrm{SE}$ at 20 m.p.m.; a few clouds; 16 inches of snow. 12 species, 363 individuals. Sharp-tailed Grouse, 16; Ring-necked Pheasant, 40; Gray Partridge, 24; Rock Dove, 16; Great Horned Owl, 2; Hairy Woodpecker, 1; Downy Woodpecker, 2; Black-billed Magpie, 14; Black-capped Chickadee, 10; Bohemian Waxwing, 6; Starling, 2; House Sparrow, 230.-Dr. A. J. Beddie, Mr. Carl Ellis, John Ellis, Mary Jane Ellis, John Horton (compiler), Mrs. Hugh McIntyre, George and Michael Rhodes, Mrs. C. V. Stokins, Mrs. F. B. Taylor, Mrs. C. West (Moose Jaw Natural History Society).

NIPAWIN, Sask. Dec. 27; 28 miles by car and 2 miles on foot in 5 hours; temp. $-5^{\circ}$; wind NW at 10 m.p.h.; overcast; 8 inches of soft snow. 14 species, 80 individuals. Pigeon Hawk, 1; Ruffed Grouse, 1; Great Horned Owl, 1; Hairy Woodpecker, 1; Downy Woodpecker, 2; Blue Jay, 2; Blackbilled Magpie, 20; Common Raven, 21; Black-capped Chickadee, 2; Boreal Chickadee, 2; Starling, 17; House Sparrow, 3; Pine Grosbeak, 6; Harris' Sparrow, 1.-David Riome (compiler), S. D. Riome, M. G. Street.

REGINA, Sask. Jan. 3, 1965; 257 miles by car and 77 miles by foot; temp. $-9^{\circ}$ to $7^{\circ}$; winds light NE at 13 m.p.h. to $S$ at 1 m.p.h.; clear, except mist over Waterfowl Park; 18 inches of snow cover in woods, most fields covered. 37 species, 3664 individuals. Mute Swan, 7;; Whistling Swan, 8; Trumpeter Swan, 1; Canada Goose, 370; Mallard, 620; Gadwall, 2; Pintail, 1; American Widgeon, 1; Lesser Scaup, 1; Common Goldeneye, 2; Prairie Falcon, 1; Pigeon Hawk, 1; Ruffed Grouse, 2; Sharp-tailed Grouse, 20; Ring-necked Pheasant, 4; Gray Partridge, 122; American Coot, 3; Rock Dove, 67; Great Horned Owl, 10; Snowy Owl, 8; Short-eared Owl, 1; Downy Woodpecker, 4; Horned Lark, 6; Black-billed Magpie, 55; Common Crow, 1; Black-capped Chickadee, 12; Robin, 1; Bohemian Waxwing, 322; 
Northern Shrike, 1; Starling, 15; House Sparrow, 1500; Purple Finch, 1; Pine Grosbeak, 2; Common Redpoll, 30; White-winged Crossbill, 3; Harris' Sparrow, 1; Snow Bunting, 458. (Add: Canvasback, 1; Ruddy Duck, 3; Sawwhet Owl 1; Hairy Woodpecker, 1)Mrs. J. B. Bailey, Fred G. Bard, Margaret Belcher, Al Binnie, Frank $H$. Brazier, Mrs. Elizabeth Cruickshank, Mr. and Mrs. Adam J. Deutscher, Elmer L. Fox, Reginald Fox, James $R$. Jowsey, Fred W. Lahrman, Ferne Lawrence, Dr. and Mrs. George F. Ledingham, Loraine McPherson, Sally Moss, Robert W. Nero (compiler), Sharon Olauson, Hugh C. Smith, Carla W. Stein, Mr. and Mirs. Herbert F. Tempel, Phil Tempel, T. Vic Wilshire (Regina Natural History Society).

ROSE VALLEY, Sask. Dec. 31; 10 miles by car and about the yard; 6 species, 103 individuals. Sharp-tailed Grouse, 15; Blue Jay, 1; Common Raven, 1; Black-capped Chickadee, 6; House Sparrow, 30; Snow Bunting, 50. (Add: Black-billed Magpie, 2, Dec. 23; Evening Grosbeak, 10, Dec. 24; Pine Grosbeak, 7, Dec. 24.)-W. A. Brownlee.

SASKATOON, Sask. Dec. $26 ; 19$ party miles on foot in $121 / 2$ party hours, and 231 miles by car in $261 / 2$ party hours; temp. $-35^{\circ}$, rising in the afternoon; overcast; wind light, 15 m.p.h. in the afternoon; 8 inches snow. 22 species, 4962 individuals. Mallard, 2; Pigeon Hawk, 1; Ruffed Grouse, 1; Sharp-tailed Grouse, 1; Ring-necked Pheasant, 16; Gray Partridge, 163; Rock Dove, 350; Great Horned Owl, 3; Hairy Woodpecker, 2; Downy Woodpecker, 4; Horned Lark, 41; Blue Jay, 7; Black-billed Magpie, 137; Common Raven, 1; Black-capped Chickadee, 27; Robin, 4; Bohemian Waxwing, 1,805; Starling, 22; House Sparrow, 2,170; Pine Grosbeak 41; Common Redpoll, 93; Snow Bunting, 71. (Add: Cedar Waxwing, 10, Dec. 27; American Goldeneye, 2, Jan. 1.)-Don Dabbs, Bob Folker, Jonathan Gerrard, $M r$. and Mrs. J. B. Gollop, Jim Hogg, Andrew Horlick, Stuart Houston (compiler), Stan Houston, Jim Hrynyk, Dr. Arygve Larsen, Mr. and Mrs. Murray Miartin, Bob Mills, Dr. Lucy Murray, Frank Roy, John and Stanley Shadick, Russ Shemko, Jim Slimmon (Saskatoon Natural History Society).
SHAUNAVON-VAL MARIE, Sask. Jan. 3, 1965; 20 miles by car; half a mile on foot; temp. $-8^{\circ}$; sunny; 20 inches of snow. 8 species, 464 individuals. Sharp-tailed Grouse, 2; Sage Grouse, 1; Ring-necked Pheasant, 2; Gray Partridge, 30; Horned Lark, 321; Black-billed Magpie, 5; House Sparrow, 100; Snow Bunting, 3. (Add: Golden Eagle, 1, Dec. 27; Marsh Hawk, 1, Dec. 26; Snowy Owl, 1, Dec. 26; Long-eared Owl, 2, Dec. 25; Downy Woodpecker, 1, Jan. 2; Starling, 1, Jan. 2.)-J. David Chandler.

SPIRIT LAKE, Sask. Jan. 2, 1965; 3 hours and 4 miles on foot about the yards and surrounding country; 10 inches of snow. 13 species, 141 individuals. Hairy Woodpecker, 8; Downy Woodpecker, 9; Gray Jay, 1; Blue Jay, 1; Black-billed Magpie, 4; Black-capped Chickadee, 40; White-breasted Nuthatch, 4; Bohemian Waxwing, 5; House Sparrow, 52; Evening Grosbeak, 5; Pine Grosbeak, 3; Common Redpoll, 7; Snow Bunting, 2.-William Anaka, Joyce Gunn.

WOLSELEY, Sask. Dec. 22; 20 miles by truck and around farmyard; temp. $-10^{\circ} ; 12$ inches of snow. 10 species, 356 individuals. Sharp-tailed Grouse, 11; Great Horned Owl, 1; Hairy Woodpecker, 1; Downy Woodpecker, 2; Horned Lark, 9; Black-billed Magpie, 16; Black-capped Chickadee, 3; House Sparrow, 100; Pine Grosbeak, 13; Snow Bunting, 200; (Add: Golden Eagle, 1; Ruffed Grouse, 1; Gray Partridge, 17; Snowy Owl, 1; Bohemian Waxwing, 19; Northern Shrike, 1; Rusty Blackbird, 2.)-Donald Hayward.

WOODROW, Sask. Dec. 22; 45 miles by truck; temp. $0^{\circ} ; 10$ to 12 inches of snow; cloudy. 10 species, 198 individuals. Sharp-tailed Grouse, 3; Ringnecked Pheasant, 7; Gray Partridge, 17; Rock Dove, 7; Snowy Owl, 1; Horned Lark, 50; Black-billed Magpie, 2; Starling, 2; House Sparrow, 9; Snow Bunting, 100.-C. H. Shulver.

YELLOW CREEK, Sask. Jan. 2, $1965 ; 21 / 2$ miles on skis, in 2 hours through farmyards, fields and light bush; temp. 15 ; wind light; mostly clear; 8 inches of snow. 5 species, 69 individuals. Gray Jay, 1; Black-billed Magpie, 2; Black-capped Chickadee, 13; House Sparrow, 51; Snow Bunting, 2. (Add: Ruffed Grouse, 1, Dec. 23; 
Great Horned Owl, 1, Dec. 28; Hairy Woodpecker, 2, Dec. 29; Downy Woodpecker, 1, Dec. 30.)- Bohdan and Irene Pylypec.

WETASKIWIN, Alta. Dec. 25; around farm yard; temp. $-18^{\circ}$ to $-10^{\circ}$; cloudy, clearing at noon; light south wind. 9 species, 41 individuals. Downy Woodpecker, 1; Northern Three-toed Woodpecker, 1; Blue Jay, 2; Black-billed Magpie, 2; Black-capped Chickadee, 6; House Sparrow, 24;
Evening Grosbeak, 3; Pine Grosbeak, 1; Common Redpoll, 1. (Add: Gray Partridge, 1, Dec. 27; Bohemian Waxwing, 20, Dec. 26; Starling, 5, Dec. 24 and 20 on Dec. 21; White-winged Croshbill, 8, Jan. 2; Snow Bunting, 125, Dec. 27.)-Fred H. Pegg.

CAMBRIDGE BAY, N.W.T. Dec. 29; 5 miles in 4 hours on foot; temp. $-50^{\circ}$; wind NW at 5; almost light. 1 species, 11 individuals. Rock Ptarmigan, 11.Ramon C. Burron.

\section{Third Annual Manitoba Christmas Bird Count, 1964}

by L. B. Smith, Winnipeg

There were 40 species seen during the count period this year by the 42 observers who braved sub-zero temperatures and a snow depth of 7-14 inches to make the annual Christmas count. Three of these species had not been recorded on the two previous counts-Long-eared Owl, Cedar Waxwing and Red-winged Blackbird. This brings the three-year total for Manitoba to 60 . Birds were reported generally to be scarce in the southern part of the province but at Thompson there were more birds than usual. Reports were received from 14 localities, two less than in 1963. All of these localities but Thompson are in the southern part of the province, ranging from Great Falls and Seven Sisters Falls in the east to Melita in the west.

The Long-eared Owl was seen at Winkler. There were two Cedar Waxwings seen in Winnipeg, one with a flock of Bohemian Waxwings and one alone. The three Red-winged Blackbirds were seen at Oak Lake in L. M. Hatch's farmyard. Black-billed Magpies were reported from nine localities, one of these being Thompson where two were seen. The numbers at the other localities ranged from one at Stonewall to 36 at Oak Lake. Blue Jays seemed to be only one third as numerous as in 1963. The highest count of Ravens was 74 from Thompson.

Snowy Owls were seen at Melita (3), Wawanesa (1) and Winnipeg (1) while one Hawk Owl was seen at Winkler. Bohemian Waxwings were seen at Boissevain (40) and at Winnipeg (132). Most of the Evening Grosbeaks were sighted in the eastern part of the province, the top number being only 36 at Seven Sisters Falls. The reports of Common Redpolls from six localities indicated they were not abundant anywhere but were well distributed throughout the province. The numbers of Snow Buntings ranged from one at Melita to 400 at Boissevain.

Some of the summer resident birds that were attempting to survive the winter were a female Mallard at Stonewall, a Golden Eagle at Oak Lake, Horned Larks at Boissevain and Wawanesa, a Robin at Winnipeg, a Slate-coloured Junco at Oak Lake and a White-throated Sparrow at Seven Sisters Falls.

I am indebted to Harold Hosford who did most of the collecting and preliminary compiling of the data. A fuller account will appear in the Newsletter of the Manitoba Natural History Society.

\section{COOPERATIVE SPRING MIGRATION STUDY}

Records are again requested for the continent-wide survey being made under the auspices of the U.S. Fish and Wildlife Service. As soon as it is available, we shall have a list of species for which reports are wanted; please write for the list and then submit your records by June 15 , 1965 , to

Mrs. STUART HOUSTON, 863 University Drive, Saskatoon, Sask. 